\title{
A Velocity-Dependent Model for Needle Insertion in Soft Tissue ${ }^{\star}$
}

\author{
Jessica R. Crouch ${ }^{1}$, Chad M. Schneider ${ }^{2}$, Josh Wainer ${ }^{2}$, \\ and Allison M. Okamura ${ }^{2}$ \\ ${ }^{1}$ Computer Science Dept., Old Dominion University, VA, USA \\ jrcrouch@odu.edu \\ 2 Engineering Research Center for Computer Integrated Surgical \\ Systems \& Technology, Johns Hopkins University, Baltimore, MD, USA \\ aokamura@jhu.edu
}

\begin{abstract}
Models that predict the soft tissue deformation caused by needle insertion could improve the accuracy of procedures such as brachytherapy and needle biopsy. Prior work on needle insertion modeling has focused on static deformation; the experiments presented here show that dynamic effects such as relaxation are important. An experimental setup is described for recording and measuring the deformation that occurs with needle insertion into a soft tissue phantom. Analysis of the collected data demonstrates the time- and velocity-dependent nature of the deformation. Deformation during insertion is shown to be well represented using a velocity-dependent force function with a linear elastic finite element model. The model's accuracy is limited to the period during needle motion, indicating that a viscoelastic tissue model may be required to capture tissue relaxation after the needle stops.
\end{abstract}

\section{Introduction}

An important source of error in needle insertion procedures such as brachytherapy and needle biopsy is the soft tissue deformation that occurs as a needle is inserted. The guidance accuracy provided by a pre-operative planning image is limited by the difference between the location of a tissue target pre-operatively and its location intra-operatively, when the target and surrounding tissues are deformed by needle insertion forces. Needle placement errors due to tissue deformation have been documented for breast biopsy [1] and prostate brachytherapy seed placement [2] 3. If the deformation caused by needle insertion could be accurately predicted, and the needle placement could be accurately controlled, the effectiveness of needle-based procedures would be improved.

Various needle insertion simulators have employed heuristic models of tissue stiffness supported by user studies but not experimental measurements of tissue deformation [4] 5]. An impediment to the development of more realistic and

\footnotetext{
* This work was supported by NSF grants EIA-0312551 and EEC-9731478, and Whitaker Foundation grant RG-02-911. The authors thank Darius Burschka for assistance with the stereo-vision system and Gregory Hager for use of his cameras.
} 
carefully validated deformable tissue models is the scarcity of data about soft tissue mechanical properties. Very limited data is available for prostate and other soft pelvic and abdominal organs. Especially lacking from the literature are experiments investigating the dynamic response of living soft tissue to interaction with needles and other surgical instruments. The instrument-tissue experiments that have been reported are limited to force recordings and do not include tissue deformation data 6, 7]. Factors making the design of such experiments challenging include acquisition speed limits for 3D imaging modalities and the difficulty of accurately extracting point displacements from soft tissue images.

Lacking force and deformation data for real tissues, prior work by DiMaio and Salcudean 8 relied on data from needle insertions into a soft tissue phantom for the development of an insertion simulation based on an elastostatic material model. Our work also relies on phantom data, but differs in that it focuses on the dynamic effects of needle insertion. Other related work includes the 2D prostate needle insertion simulation developed by Alterovitz, et al. [9], and the Truth Cube developed by Kerdok, et al. 10. Our tissue phantom's design was inspired by the Truth Cube, a silicone gel volume with embedded fiducials whose displacements were tracked to estimate the gel's properties in compression tests.

\section{Experiment Design}

An 18 gauge diamond-tip brachytherapy needle was inserted at a constant velocity into a tissue phantom impregnated with a grid of tiny fiducials for each experiment trial. Needle forces were recorded for insertion velocities from 3 to 21 $\mathrm{mm} / \mathrm{s}$. The phantom deformation was monitored by optically tracking the fiducial displacements. The experiment hardware included a specially constructed tissue phantom, a device that precisely controlled needle motion, a force/torque sensor, and calibrated stereo video cameras for recording fiducial motion.

\subsection{Tissue Phantom}

The tissue phantom was made of GE RTV-6166, the same transparent, homogeneous silicone gel used for the Truth Cube [10]. An experienced brachytherapy surgeon selected this gel as providing more realistic resistance to a needle than alternative soft plastic and porcine gelatin materials.

The phantom was constructed by pouring gel into an acrylic box in $8 \mathrm{~mm}$ layers, placing it in a vacuum chamber to remove air bubbles, and then dropping a row of $0.8 \mathrm{~mm}$ fiducials onto the surface as the gel began to set. Subsequent layers were added before curing completed so that boundaries did not form between layers. The needle was inserted perpendicular to the plane of the layers, so variations in stiffness caused by the layer construction would have caused periodic irregularities in the force data corresponding to the layer width. Layer effects were deemed negligible because such irregularities were not detected.

The fiducials' small size minimizes their influence on the material properties of the phantom tissue. Kerdok performed indentation tests on samples of RTV-6166 with and without implanted fiducials and reported that there was no 
discernible change in its material properties even when using fiducials that had nearly twice the diameter of the ones used in our work 10.

\subsection{Needle Insertion Device}

The needle was inserted using an encoded Maxon A-max 22 DC motor connected to a translational stage via a capstan drive. Constant velocity during insertion was maintained using a proportionalderivative controller for the needle stage position. A 6-axis force/torque sensor (Nano17 from ATI Industrial Automation) was mounted at the needle base. Needle forces, position, and velocity were recorded at 500 $\mathrm{Hz}$. The phantom was attached to the needle insertion device as shown in Fig. 1 so that the needle was aligned with the plane of fiducials.

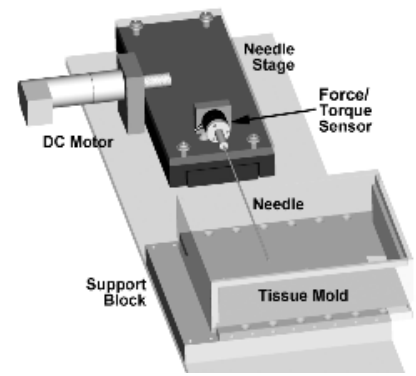

Fig. 1. Tissue phantom was $113 \mathrm{~mm}$ $\times 135 \mathrm{~mm} \times 30 \mathrm{~mm}$; the fiducial grid has $5 \mathrm{~mm} \times 8 \mathrm{~mm}$ spacing

\subsection{Fiducial and Needle Tracking}

Insertions were recorded by two Sony DFW-X700 digital cameras at $7.5 \mathrm{~Hz}$ and $640 \times 480$ resolution. A $9 \times 9$ grid of fiducials was tracked by convolving each captured image with a rotationally symmetric Laplacian of Gaussian (LoG) kernel that was width matched to the fiducials. The maximum match near each fiducial was tagged as the fiducial's center. Tracking results are shown in Fig. 2.

The needle trajectory was found by convolving one image from each trial with a LoG kernel that was width matched to the needle diameter, selecting the peak match points, and performing a least squares fit of a line to the match point coordinates. Needle tip tracking was performed using images that were masked to include only the portion of the image near the needle trajectory. Tracking was accomplished by computing difference images between successive video frames. The needle's stripes caused bright bands in the difference images when the needle was shifted; the leading band indicated the needle tip position.

\subsection{D Reconstruction and Error Estimation}

The fiducial and needle tracking algorithms provide pairs of corresponding image coordinates from the right and left camera views. From these, 3D coordinates
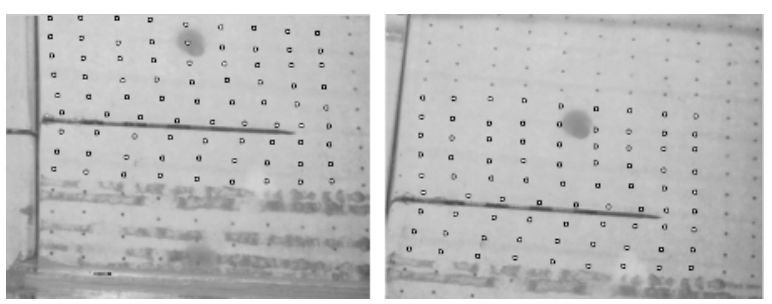

Fig. 2. Both camera views are shown with tracked fiducials marked by black circles. Untracked peripheral fiducials appear fainter. The fiducials and needle are clearly visible because the transparent gel is backlit. 
were computed using a standard computer vision algorithm that relies on stereo camera calibration parameters [11. The error present in the 3D coordinates can be attributed to uncertainty in the camera calibration and to limitations in point tracking accuracy. This error can be estimated by examining the coordinates computed for the needle tip. Since the needle path is known to be a straight line (there is no needle bending, as verified by visual and force data inspection), the deviation of the 3D tip coordinates from a line is an indication of the error. To investigate this, a least squares line fit was performed for the $3 \mathrm{D}$ needle tip coordinates of nine trials. The mean distance between the tip coordinates and the line was $0.73 \mathrm{~mm}$ with a standard deviation of $1.23 \mathrm{~mm}$. The largest component of the error was along the camera's viewing direction, with a mean of $0.65 \mathrm{~mm}$ and standard deviation of $1.10 \mathrm{~mm}$ in that direction.

\section{$3 \quad$ Force and Deformation Data}

\subsection{Insertion Force}

Needle forces were recorded for three insertions at each of the following velocities: $3,6,9,12,15,18$, and $21 \mathrm{~mm} / \mathrm{s}$. Fig. 3 shows total needle force versus insertion depth for all trials, demonstrating a velocity-dependent effect. Fig. 4 graphs the slopes of the curves in Fig. 3 vs. insertion velocity. As shown, a good approximation to the force function is provided by a scaled and shifted log function.

\subsection{Force Decay}

A gradual reduction in force after the needle halts was another dynamic effect observed. Fig. 5 shows the force decaying for 500 seconds after the needle halts. The decay can be analyzed using the following time dependent function:

$$
f_{i}=\left(1-\alpha_{i}\right) * f_{i-1}
$$

Fig. 3. Total needle force vs. needle insertion depth for different insertion velocities

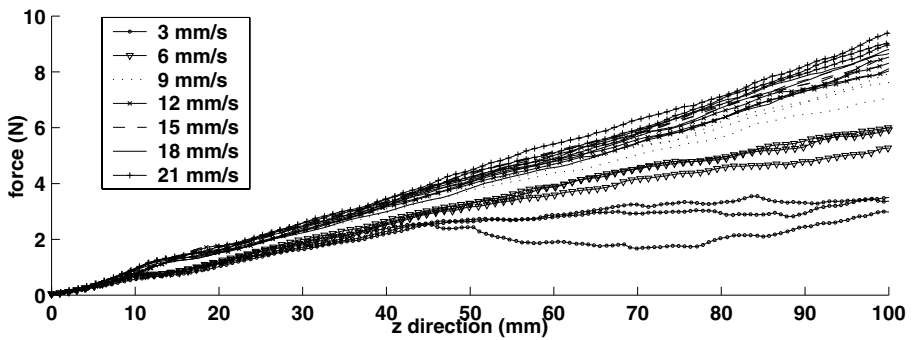

Fig. 4. Slope of force vs. position for different insertion speeds is shown by the solid line. The observed slope data is averaged across three trials for each speed, and standard deviations are shown. The dotted line shows the best fit scaled and shifted log function.

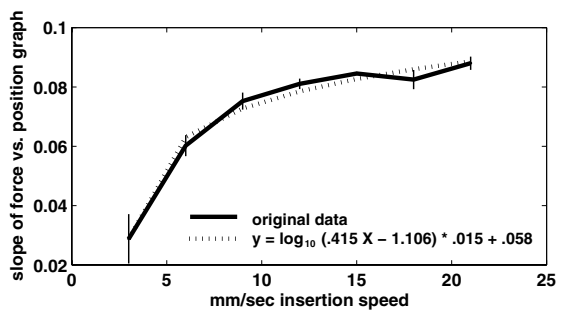


$\alpha_{i}$ represents the fraction of force that dissipates each time step. The $\alpha_{i}$ values in Fig. 5 were computed for the recorded force data. The shape of the $\alpha$ curves suggested a good fit might be obtained using Gaussian functions. Thus, the following function models $\alpha$ :

$$
\begin{aligned}
\alpha(f)=h_{\alpha} * G(f, \sigma, \mu) \quad \text { where } & =\left(c_{1} * \max (f)\right)+c_{2} \\
\mu & =\left(c_{3} * \max (f)\right)+c_{4} \\
h & =c_{5} * \sigma \\
G(x, \sigma, \mu) & =\frac{1}{\sigma \sqrt{2 \pi}} e^{\frac{-(x-\mu)^{2}}{2 \sigma^{2}}} \quad \text { (Gaussian eqn.) }
\end{aligned}
$$

$f$ is the needle force. Optimizing this model to fit the recorded data yielded constant values: $c_{1}=0.1267 \quad c_{2}=0.7613 \quad c_{3}=0.8171 \quad c_{4}=0.8194 \quad c_{5}=2.15$. The force decay model with these constants is shown in Fig. 6. The difference between recorded forces and model forces was at all points less than $0.5 \mathrm{~N}$.
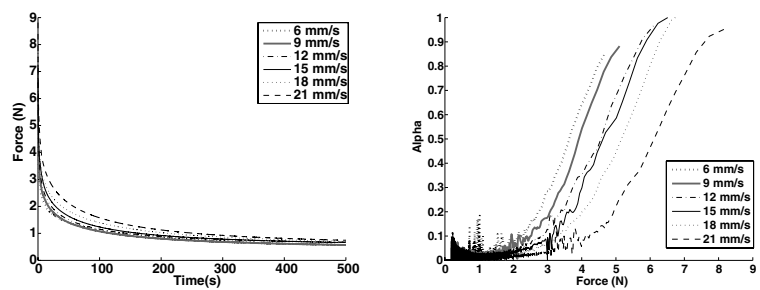

Fig. 5. Left: 500 sec. of recorded needle force, showing force decay after needle halts. Right: $\alpha$ parameter computed for the recorded needle force data
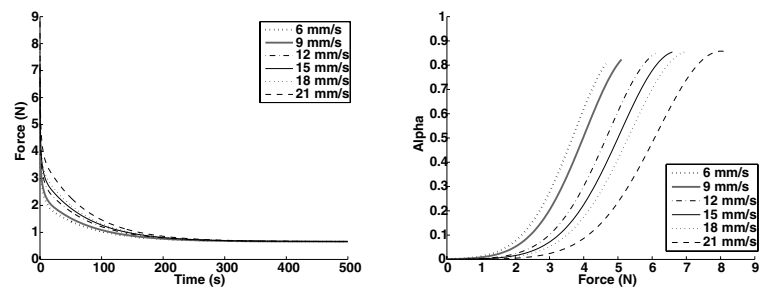

Fig. 6. Left: 500 sec. of model generated needle force, showing force decay after needle halts. Right: $\alpha$ parameter computed for the model generated force data

\subsection{Needle Shaft Force Distribution}

The simplest force distribution is a constant force level defined by the slope of the appropriate curve from Fig. 3. To investigate the validity of this simple model, shaft force constants defined by the equation in Fig. 4 were integrated along the length of the needle shaft for trials at different insertion speeds. The results of the integration shown in Fig. 7 closely match the measured needle forces, supporting a generally flat force distribution model.

To further examine the distribution of needle force, an optimization approach was applied in conjunction with a finite element model. Using the recorded experiment data, the fidelity of a needle model can be judged based on the accuracy with which it predicts fiducial motion. For the optimization, needle force models were represented by piecewise cubic splines. Two spline segments were allocated for $3 \mathrm{~mm}$ near the tip, and one segment was allocated to each 10 $\mathrm{mm}$ along the rest of the needle shaft. The spline functions represented the force 
magnitude; consistent with the data recorded from the force/torque sensor, the force direction was assumed to be the insertion direction.

The finite element model shown in Fig. 8 was constructed with Femlab (COMSOL, Inc.). A linear elastic material model was applied, with a Young's modulus of 14.9 $\mathrm{kPa}$ based on the work by Kerdok 10, and a Poisson's ratio of 0.49 to indicate near incompressibility. Fixed boundary conditions were applied to the phantom sides held stationary by the acrylic casing. Given a force distribution, finite element analysis yielded the deformation and fiducial displacements, as shown

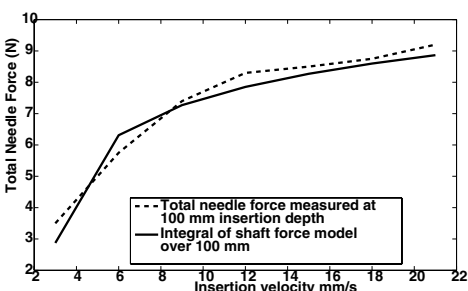

Fig. 7. Force vs. needle insertion velocity at $100 \mathrm{~mm}$ needle insertion integrated constant model force across all insertion in Fig. 9.

A quasi-Newton optimization method was applied to the force distribution with the objective of minimizing the error in the predicted fiducial displacements. A portion of the optimization results are shown in Fig. 10. Though noisy, the optimized distributions indicate relatively constant force along the needle shaft followed by a dip and pronounced peak at the tip. The force peak is required to overcome the material's resistance to cutting/crack propagation. The dip may be due to a snap-back effect that occurs as compressed tissue at the tip fractures and relaxes. The mean error in the predicted fiducial motion was $0.31 \mathrm{~mm}$, with a standard deviation of $0.21 \mathrm{~mm}$. Optimization was performed over a period of two weeks using a PC workstation with two $3.2 \mathrm{GHz}$ Intel Xeon processors.

Based on these results, the following time and velocity-dependent function for needle force was constructed to fit the experiment data:
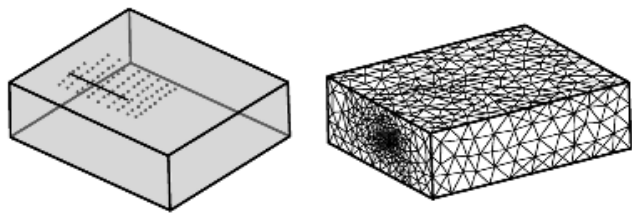

Fig. 8. Femlab phantom tissue model and tetrahedral mesh with higher resolution around the needle
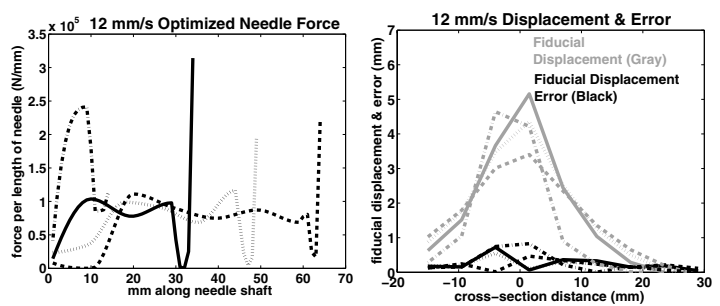

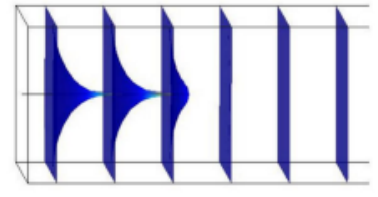

Fig. 9. Slices through the deformed finite element model

Fig. 10. Left: optimized distributions for 4 time points during insertion at $12 \mathrm{~mm} / \mathrm{s}$. Right: deformation in a slice perpendicular to the needle. In gray, the magnitude of recorded fiducial displacements; in black, the displacement prediction error. 


$$
f_{i}(x, v)= \begin{cases}2 * \text { shaftForce } & \text { if } v>0, x<1 \\ 0.5 * \text { shaftForce } & \text { if } v>0,1 \leq x<2 \\ \text { shaftForce } & \text { if } v>0, x \geq 2 \\ f_{i-1} *\left(1-\alpha_{i} * \Delta t\right) & \text { if } v=0\end{cases}
$$

where

$$
\text { shaftForce }=\log (0.415 * v-1.106) * 0.015+0.058
$$

(see Fig.4)

Here $x$ is distance from the needle tip, $v$ is insertion velocity, $i$ is time step index, $\alpha_{i}$ is defined by Eqn. 22, and $\Delta t$ is time step length. $f_{i}$ defines the force magnitude applied in the direction of needle insertion. Constants in Eqn. 3 will vary depending on the selection of needle and material.

The needle force function was constructed to satisfy these observations:

- The needle force depends on insertion velocity, as shown in Figs. 3 and 4.

- The majority of the needle force is evenly distributed along the shaft because: (1) for constant velocity insertion the force increases at a nearly constant rate (see Fig. 3) and (2) assuming a flat force distribution results in a total needle force that closely matches the measured force (see Fig. 7).

- Optimization indicated a force peak at the tip preceded by a small force dip.

- When the needle halts, the force decays according to Eqns. 1 and 2.

\section{Results}

Force profiles were generated by the model given in Eqn. 3 and were applied to the Femlab 3D finite element model. Some of the force profiles generated and cross-sections of the corresponding fiducial displacements are shown in Fig. 11 The results indicate this model produces an excellent approximation to the forces and deformations recorded in the experiment during the active insertion phase. When the needle halts, the model produces force relaxation that closely match the decay in needle force recorded in the experiment (see Fig. 4). However, the deformation produced by the model in the relaxation phase does not match the recorded deformation as well. As shown in Fig. 11, the recorded deformation decayed more quickly than the recorded force after the needle stopped.

\section{Conclusions and Future Work}

Through experimental observation and FEM modeling, this work has shown that a static linear elastic tissue model combined with a dynamic force function can accurately model forces and deformations during insertion at varying speeds. However, the accuracy of the model diminishes during the relaxation phase after the needle halts because real and phantom soft tissues are viscoelastic [12. A viscoelastic model, to be considered in future work, might account for differing rates of force and deformation decay, consistent with the results shown Fig. 11.

It is not ideal that phantoms are used rather than in vivo tissues, but this is necessary for repeatability and validation. Thus, future work may include tests on real tissues, using a modified experimental setup. By using bi-plane x-ray 

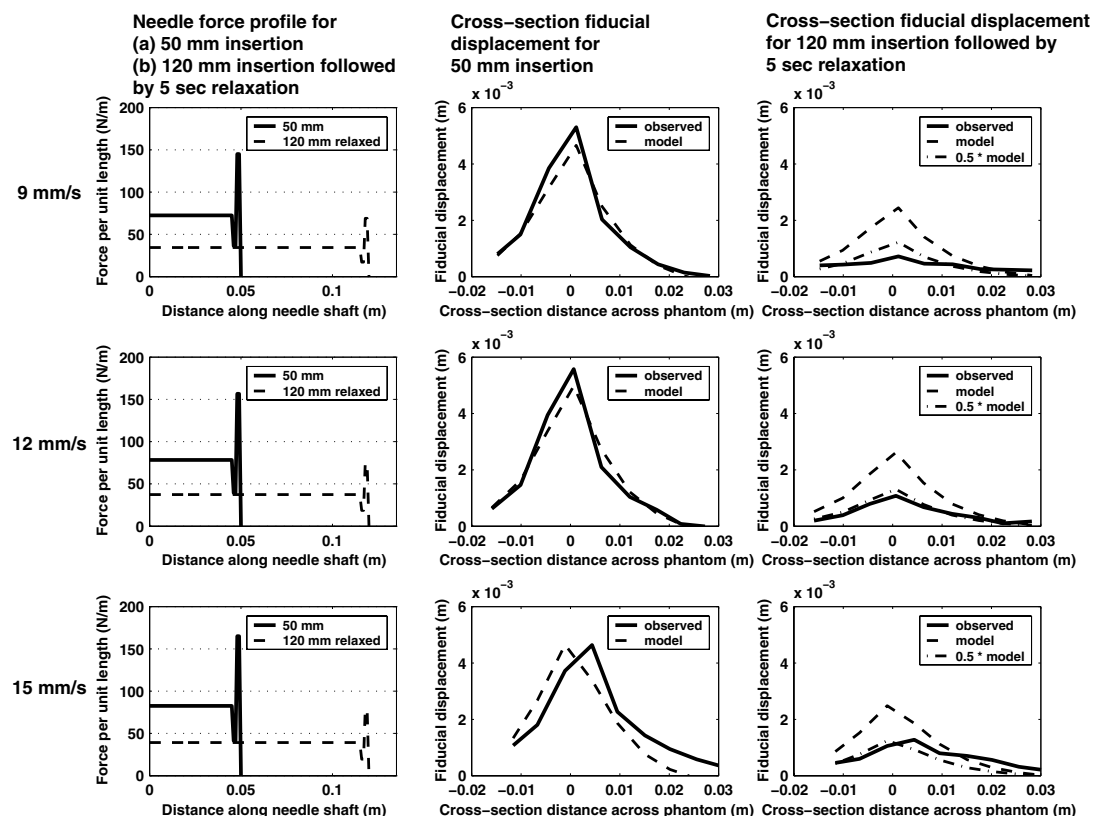

Fig. 11. Column 1: model force profile for $50 \mathrm{~mm}$ insertion without relaxation and 120 mm insertion with $5 \mathrm{sec}$. relaxation. Column 2: model and experiment displacements for $50 \mathrm{~mm}$ insertion. Column 3: model and experiment displacements for $120 \mathrm{~mm}$ insertion with 5 sec. relaxation; 0.5 times model displacements is also shown.

to track radio-opaque fiducials or tissue features, the experimental methodology could be applied to non-transparent tissue samples. The effect of perfusion will likely impact on a tissue's dynamic response [13. Further experiments should include many repetitions to allow statistical measures of model accuracy.

\section{References}

1. Deurloo, E., Gilhuijs, K., Kool, L., Muller, S.: Displacement of breast tissue and needle deviations during stereotactic procedures. Invest. Rad. 36 (2001) 347-353

2. Roberson, P.L., Narayana, V., McShan, D.L., et al: Source placement error for permanent implant of the prostate. Medical Physics 24 (1997) 251-257

3. Nath, S., Chen, Z., Yue, N., Trumpore, S., Peschel, R.: Dosimetric effects of needle divergence in prostate seed implant using $125 \mathrm{I}$ and $103 \mathrm{Pd}$ radioactive seeds. Medical Physics (2000) 1058-1066

4. Xuan, J., Wang, Y., Sesterhenn, I., Mou, J., Mun, S.: 3-D model supported prostate biopsy simulation and evaluation. MICCAI 1496 (1998) 358-367

5. Gorman, P., Krummel, T., Webster, R., Smith, M., Hutchens, D.: A prototype haptic lumbar puncture. Proc of Medicine Meets Virtual Reality (2000) 106-109

6. Brouwer, I., Ustin, J., Bentley, L., et al: Measuring in vivo animal soft tissue properties for haptic modeling in surgical simulation. Medicine Meets Virtual Reality (2001) 69-74 
7. Okamura, A.M., Simone, C., O'Leary, M.D.: Force modeling for needle insertion into soft tissue. IEEE Transactions on Biomedical Engineering (2004) 1707-1716

8. DiMaio, S.P., Salcudean, S.E.: Needle insertion modelling and simulation. IEEE Trans on Robotics and Automation: Special Issue on Medical Robotics (2003)

9. Alterovitz, R., Pouliot, J., Taschereau, R., Hsu, I., Goldberg, K.: Needle insertion and radioactive seed implantation in human tissues: Simulation and sensitivity analysis. Proc. IEEE Intl Conference on Robotics and Automation (2003)

10. Kerdok, A.E., Cotin, S.M., Ottensmeyer, M.P., Galea, A., Howe, R.D., Dawson, S.L.: Truth cube: Establishing physical standards for real time soft tissue simulation. Medical Image Analysis 7 (2003) 283-291

11. Forsyth, D., Ponce, J.: Computer Vision, A Modern Approach. Prentice Hall, Upper Saddle River, New Jersey (2003)

12. Fung, Y.C.: Biomechanics: Mechanical Properties of Living Tissues. Springer, New York (1993)

13. Ottensmeyer, M.P., Kerdok, A.E., Howe, R.D., Dawson, S.L.: The effects of testing environment on the viscoelastic properties of soft tissues. Second International Symposium on Medical Simulation (2004) 9-18 